Portland State University

PDXScholar

1989

\title{
Analytically Reduced Form Of Multicenter Integrals From Gaussian Transforms
}

Jack C. Straton

Portland State University, straton@pdx.edu

Follow this and additional works at: https://pdxscholar.library.pdx.edu/phy_fac

Part of the Physics Commons

Let us know how access to this document benefits you.

\section{Citation Details}

Straton, J. C. (1989). Analytically reduced form of multicenter integrals from Gaussian transforms. Physical Review A, 39(4), 1676.

This Article is brought to you for free and open access. It has been accepted for inclusion in Physics Faculty Publications and Presentations by an authorized administrator of PDXScholar. Please contact us if we can make this document more accessible: pdxscholar@pdx.edu. 


\title{
Analytically reduced form of multicenter integrals from Gaussian transforms
}

\author{
Jack C. Straton* \\ Laboratory for Astronomy and Solar Physics, Goddard Space Flight Center, \\ National Aeronautics and Space Administration, Greenbelt, Maryland 20771 \\ (Received 17 August 1988)
}

\begin{abstract}
In a previous paper the analytically reduced form was found for the general class of integrals containing multicenter products of $1 s$ hydrogenic orbitals, Coulomb or Yukawa potentials, and plane waves. The method consisted of combining all angular dependence within a single quadratic form by means of a three-dimensional Fourier transform and a one-dimensional Feynman transform for each term in the product and an additional integral transformation to move the resulting denominator into an exponential to be summed with the vector products in the plane waves. This quadratic form was then diagonalized with respect to the (introduced) momentum integrals and diagonalized again with respect to the (original) spatial integrals. In the present paper the four-dimensional Fourier-Feynman transformations are replaced by the one-dimensional Gaussian transformation so that only one diagonalization is required, yielding a simpler reduced form for the integral. The present work also extends the result to include all $s$ states and pairs of states with $l \neq 0$ summed over the $m$ quantum number.
\end{abstract}

\section{INTRODUCTION}

A large class of problems in atomic and molecular physics depends on the evaluation of integrals composed of a product of plane waves with hydrogenic orbitals and Coulomb or Yukawa potentials in which the arguments are arbitrary linear combinations of the coordinates of integration and any external coordinates,

$$
\begin{aligned}
& S_{\mu_{1} \cdots \mu_{N}}^{\lambda_{1} \cdots \lambda_{N} \eta_{1} \cdots \eta_{M}}\left(\mathbf{p}_{1}, \mathbf{p}_{2}, \ldots, \mathbf{p}_{m} ; \mathbf{y}_{1}, \mathbf{y}_{2}, \ldots, \mathbf{y}_{N+M}\right) \\
& \quad=\int d^{3} x_{1} \cdots d^{3} x_{m} e^{-i\left(\mathbf{p}_{1} \cdot \mathbf{x}_{1}+\cdots+\mathbf{p}_{m} \cdot \mathbf{x}_{m}\right)} u_{\mu_{1}}^{\lambda_{1}}\left(\mathbf{R}_{1}\right) \cdots u_{\mu_{N}}^{\lambda_{N}}\left(\mathbf{R}_{N}\right) V_{\eta_{1}}\left(\mathbf{R}_{N+1}\right) \cdots V_{\eta_{M}}\left(\mathbf{R}_{N+M}\right)
\end{aligned}
$$

where $\mu_{i}$ are quantum numbers,

$$
\begin{aligned}
& \mathbf{R}_{i}=\sum_{j=1}^{m} t_{i j} \mathbf{x}_{j}+\sum_{j=1}^{N+M} u_{i j} \mathbf{y}_{j}, \\
& V_{\eta}(\mathbf{R})=\frac{e^{-\eta R}}{R}
\end{aligned}
$$

and

$$
R \equiv(\mathbf{R} \cdot \mathbf{R})^{1 / 2}
$$

The $1 s$ hydrogenic wave function (in atomic units) is, for example,

$$
u_{1 s}^{\lambda}(\mathbf{R})=\frac{\lambda^{3 / 2}}{\sqrt{\pi}} e^{-\lambda R}
$$

where

$$
\lambda_{j}=\frac{Z_{j}}{a_{0 j}} .
$$

Direct integration of the spatial representation (1) is generally not possible because the angular dependence of each variable of integration appears in several of the square roots of quadratic forms in functions such as (3) and (5), in addition to the vector products in the plane waves.
Gaussian-type orbitals ${ }^{1}$ (GTO's) are used in some applications $^{2}$ to approximate hydrogenic orbitals because the quadratic arguments in these exponentials may simply be added. The drawback of these functions is that they do not represent the correct wave function near the nuclear centers nor at large distances. Furthermore, in scattering problems the use of approximate wave functions introduces discrepancies between post and prior results that would not exist when exact wave functions are used. ${ }^{3}$

The more common analytical technique in reducing such matrix elements to tractable numerical form has been to Fourier transform ${ }^{4-6}$ parts of the integrand, producing denominators that are (powers of) simple quadratic forms for both orbitals and potentials,

$$
u_{1 s}^{\lambda}(\mathbf{R})=\left[\frac{\lambda}{\pi}\right]^{5 / 2} \int d^{3} k \frac{e^{i \mathbf{k} \cdot \mathbf{R}}}{\left(\lambda^{2}+k^{2}\right)^{2}},
$$

and $^{7}$

$$
V_{\eta}(\mathbf{R})=\frac{1}{2 \pi^{2}} \int d^{3} k \frac{e^{i \mathbf{k} \cdot \mathbf{R}}}{\left(\eta^{2}+k^{2}\right)}, \quad \eta \geq 0 .
$$

Because of this structure, the angular dependence of the denominators may be combined by introducing Feynman integral transforms for the denominators, ${ }^{8,9}$ generalized to allow arbitrary powers of the denominators, ${ }^{10,11}$ 


$$
\frac{1}{D_{1}^{1+m_{1}} D_{2}^{1+m_{2}} \cdots D_{n}^{1+m_{n}}}=\frac{(N-1) !}{\left(m_{1}\right) !\left(m_{2}\right) ! \cdots\left(m_{n}\right) !} \int_{0}^{1} d \alpha_{1} \int_{0}^{1} d \alpha_{2} \cdots \int_{0}^{1} d \alpha_{n} \alpha_{1}^{m_{1}} \alpha_{2}^{m_{2}} \cdots \alpha_{n}^{m_{n}} \frac{\delta\left[1-\sum_{i=1}^{n} \alpha_{i}\right.}{\left[\sum_{i=1}^{n} \alpha_{i} D_{i}\right]^{N}}
$$

where $N=n+\sum_{i} m_{i}$. If the product of functions includes plane waves, as shown in two recent papers, ${ }^{10,12}$ an additional integral transform may be introduced, ${ }^{13}$

$$
(N-1) ! D^{-N}=\int_{0}^{\infty} d \rho \rho^{N-1} e^{-\rho D},
$$

to allow the angular dependence of the resulting denominator to be combined with the angular dependence of the plane waves in a single quadratic form within an exponential. Finally, an orthogonal transformation ${ }^{14}$ may be invoked to diagonalize the resulting quadratic form, first with respect to momentum variables and then with respect to spatial variables, allowing integrals over all of these variables to be evaluated.

This process is very effective in reducing the dimensionality of the original integral (1) from a $3 m$ dimensional integral to an $(N+M-1)$-dimensional Feynman integral (where $m \leq N+M$ ). In fact, the final result has been given for the entire class of integrals in which the orbitals are in the ground state. ${ }^{12}$ However, the Gaussian transformation of Sec. II achieves the same result with considerably less work and is easily generalized to higher $s$ states. Section III presents the technique for diagonalizing the spatial dependence and gives the reduced form of (1) for ground-state wave functions. Examples are given in Sec. IV and the extensions to excited states are given in Sec. V.

\section{GAUSSIAN TRANSFORM}

Instead of introducing the Fourier transform, a threedimensional momentum integral, and a one-dimensional Feynman integral for each function in (1), one may introduce the one-dimensional integral transformation for the potentials $^{15}$

$$
\frac{e^{-\lambda R}}{R}=\frac{1}{\sqrt{\pi}} \int_{0}^{\infty} d \rho \frac{e^{-R^{2} \rho-\lambda^{2} / 4 \rho}}{\rho^{1 / 2}},
$$

to utilize the quadratic (Gaussian) coordinate dependence, or the transformation first suggested by Kikuchi ${ }^{16}$ for this purpose,

$$
\frac{e^{-\lambda R}}{R}=\frac{2}{\sqrt{\pi}} \int_{0}^{\infty} d \rho e^{-R^{2} \rho^{2}-\lambda^{2} / 4 \rho^{2}},
$$

where in both cases

$$
R>0 \text { and } \lambda \geq 0 \text {. }
$$

The transformation that is regular at the origin, ${ }^{17}$ introduced by Shavitt and Karplus, ${ }^{18}$ is

$$
e^{-\lambda R}=\frac{\lambda}{2 \sqrt{\pi}} \int_{0}^{\infty} d \rho \frac{e^{-R^{2} \rho-\lambda^{2} / 4 \rho}}{\rho^{3 / 2}},
$$

or $^{19}$

$$
e^{-\lambda R}=\frac{\lambda}{\sqrt{\pi}} \int_{0}^{\infty} d \rho \frac{e^{-R^{2} \rho^{2}-\lambda^{2} / 4 \rho^{2}}}{\rho^{2}},
$$

where in both cases

$$
R \geq 0 \text { and } \lambda>0 \text {. }
$$

Then the hydrogenic $1 s$ wave functions transform as

$$
u_{1 s}^{\lambda}(\mathbf{R})=\frac{\lambda^{5 / 2}}{2 \pi} \int_{0}^{\infty} d \rho \frac{e^{-R^{2} \rho-\lambda^{2} / 4 \rho}}{\rho^{3 / 2}},
$$

or

$$
u_{1 s}^{\lambda}(\mathbf{R})=\frac{\lambda^{5 / 2}}{\pi} \int_{0}^{\infty} d \rho \frac{e^{-R^{2} \rho^{2}-\lambda^{2} / 4 \rho^{2}}}{\rho^{2}} .
$$

Other powers of $R$ multiplying the left-hand side of (11) may be generated by differentiation,

$$
R^{j-1} e^{-\beta R}=(-1)^{j} \frac{\partial^{j}}{\partial \beta^{j}} \frac{1}{\sqrt{\pi}} \int_{0}^{\infty} d \rho \frac{e^{-R^{2} \rho-\beta^{2} / 4 \rho}}{\rho^{1 / 2}}
$$

Let

$$
x=\frac{\beta}{2 \sqrt{\rho}},
$$

then ${ }^{20}$

$$
\begin{aligned}
(-1)^{j} \frac{\partial^{j}}{\partial \beta^{j}} e^{-\beta^{2} / 4 \rho} & =\frac{1}{2^{j} \rho^{j / 2}} e^{-x^{2}}\left((-1)^{j} e^{x^{2}} \frac{\partial^{j}}{\partial x^{j}} e^{-x^{2}}\right) \\
& =\frac{1}{2^{j} \rho^{j / 2}} e^{-\beta^{2} / 4 \rho} H_{j}\left[\frac{\beta}{2 \sqrt{\rho}}\right],
\end{aligned}
$$

where $H_{j}$ is a Hermite polynomial. This gives the result $^{21}$ found by Wright, ${ }^{22}$

$$
R^{j-1} e^{-\beta R}=\frac{1}{2^{j} \sqrt{\pi}} \int_{0}^{\infty} d \rho \frac{e^{-R^{2} \rho-\beta^{2} / 4 \rho}}{\rho^{(j+1) / 2}} H_{j}\left(\frac{\beta}{2 \sqrt{\rho}}\right)
$$

where the coefficient is sometimes misquoted m $^{23,24}$ as $1 /(2 j \sqrt{\pi})$...

In (20) the quadratic form that contains the spatial dependence, $\exp \left(-R^{2} \rho\right)$, is sequestered from the part of the shape function, the remainder of the integrand, that depends on $j$. For this reason the radial part of excited states may be included with ease. In contrast, the Fourier-Feynman transformation requires derivatives of $D$ in (10) with respect to the various $\lambda$ 's of any excited states because $\lambda$ and the quadratic form $k^{2}$ are tied together in the equivalent of (7). So the general reduced 
form of (1), for $s$ states, obtained through Gaussian transformation, is simply an integral with an algebraic integrand. This result is far easier to use than the reduced form obtained using Fourier-Feynman transformation in which the integrand contains derivatives still to be taken in each application of the general form.

The Gaussian transform was put on a practical footing by Taylor, ${ }^{25}$ who developed a systematic procedure for obtaining the shape function $f(t)$ of the Gaussian transform,

$$
u(R) \equiv G[f(t) ; R]=\int_{0}^{\infty} d t e^{-R^{2} t} f(t),
$$

from a given object function $u(R)$ through the introduction of an inverse transform

$$
f(t) \equiv G^{-1}[u(R) ; t] \text {. }
$$

This is possible because, as pointed out in the original paper by $\mathrm{Kikuchi}^{16}$ and in more detail by Bishop and Somorjai, ${ }^{26}$ the Gaussian transform is a modification of the Laplace transform obtained from (21) by the replacement

$$
s=R^{2} .
$$

Thus one may exploit the extensive tables of Laplace transforms and inverse Laplace transforms, ${ }^{27-29}$ to find

$$
u(r)=G[f(t) ; R] \equiv L\left[f(t) ; R^{2}\right]
$$

and

$$
f(t)=G^{-1}[u(R) ; t]=L^{-1}\left[u\left(s^{1 / 2}\right) ; t\right] .
$$

In fact, $(20)$ is given by setting $u(R)$ of $\left(22^{\prime}\right)$ equal to the left-hand side of (17). ${ }^{30}$ With this structure in place, the alternate transformations (12), (14), and (16) will not be of extensive use.

\section{DIAGONALIZING THE SPATIAL QUADRATIC FORM}

With the theory of the Gaussian transform in place, the reduced form of (1) in the special case where each atom is in the ground state may now be found. Using (11) and (15), one may immediately write all of the spatial dependence within a single quadratic form,

$$
\begin{aligned}
& S_{1 s_{1} \cdots \lambda_{N}}^{\lambda_{1} \cdots \lambda_{N} \eta_{1} \cdots \eta_{M}}\left(\mathbf{p}_{1}, \mathbf{p}_{2}, \ldots, \mathbf{p}_{m} ; \mathbf{y}_{1}, \mathbf{y}_{2}, \ldots, \mathbf{y}_{N+M}\right) \\
& =\frac{\left(\lambda_{1} \cdots \lambda_{N}\right)^{5 / 2}}{2^{N} \pi^{N+M / 2}} \\
& \quad \times \int d^{3} x_{1} \cdots d^{3} x_{m} \\
& \quad \quad \times \int_{0}^{\infty} d \rho_{1} \cdots d \rho_{N+M} \frac{\exp \left(-\lambda_{1}^{2} / 4 \rho_{1}-\cdots-\lambda_{N}^{2} / 4 \rho_{N}-\eta_{1}^{2} / 4 \rho_{N+1}-\cdots-\eta_{M}^{2} / 4 \rho_{N+M}\right)}{\left(\rho_{1} \cdots \rho_{N}\right)^{3 / 2}\left(\rho_{N+1} \cdots \rho_{N+M}\right)^{1 / 2}} e^{-Q}
\end{aligned}
$$

where

$$
\begin{aligned}
Q & =i\left(\mathbf{p}_{1} \cdot \mathbf{x}_{1}+\cdots+\mathbf{p}_{m} \cdot \mathbf{x}_{m}\right)+\rho_{1} R_{1}^{2}+\cdots+\rho_{N+M} R_{N+M}^{2} \\
& \equiv \mathbf{V}^{T} \mathbf{W} \mathbf{V} .
\end{aligned}
$$

In the latter compact notation,

$$
\begin{aligned}
& \mathbf{V}^{T}=\left(\mathbf{x}_{1}, \mathbf{x}_{2}, \ldots, \mathbf{x}_{m}, 1\right), \\
& \mathbf{W}=\left(\begin{array}{ccccc}
a_{11} & a_{12} & \cdots & a_{1 m} & \mathbf{b}_{1} \\
a_{21} & a_{22} & \cdots & a_{2 m} & \mathbf{b}_{2} \\
\vdots & \vdots & & \vdots & \vdots \\
a_{m 1} & a_{m 2} & \cdots & a_{m m} & \mathbf{b}_{m} \\
\mathbf{b}_{1} & \mathbf{b}_{2} & \cdots & \mathbf{b}_{m} & C
\end{array}\right), \\
& a_{i j}=\sum_{k=1}^{N+M} \rho_{k} t_{k i} t_{k j}, \\
& \mathbf{b}_{i^{\prime}}=\frac{i \mathbf{p}_{i^{\prime}}}{2}+\sum_{k=1}^{N+M} \rho_{k} t_{k i^{\prime}} \sum_{j=1}^{N+M} u_{k j} \mathbf{y}_{j},
\end{aligned}
$$

and

$$
C=\sum_{k=1}^{N+M} \sum_{j=1}^{N+M} \sum_{j^{\prime}=1}^{N+M} \rho_{k} u_{k j} u_{k j^{\prime}} \mathbf{y}_{j} \cdot \mathbf{y}_{j^{\prime}} .
$$

Now suppose one can find an orthogonal transformation that reduces $Q$ to diagonal form,

$$
Q^{\prime}=a_{1}^{\prime} x_{1}^{\prime 2}+\cdots+a_{m}^{\prime} x_{m}^{\prime 2}+c^{\prime},
$$

where, as shown by Chisholm, ${ }^{14}$ the $a_{j}^{\prime}$ are positive. Then after a simple translation in $\left\{\mathbf{x}_{i}, \mathbf{x}_{2}, \ldots, \mathbf{x}_{m}\right\}$ space (with Jacobian $=1$ ), the $x^{\prime}$ integrals may be done using

$\int d^{3} x_{1}^{\prime} \cdots d^{3} x_{m}^{\prime} e^{-\left(a_{1}^{\prime} x_{1}^{\prime 2}+\cdots+a_{m}^{\prime} x_{m}^{\prime 2}\right)}=\left(\frac{\pi^{m}}{\Lambda^{\prime}}\right)^{3 / 2}$

where

$$
\Lambda^{\prime}=a_{1}^{\prime} a_{2}^{\prime} \cdots a_{m}^{\prime}
$$

But the orthogonal transformation leading to (32) leaves the determinants

$$
\Omega=\operatorname{det} W
$$


and

$$
\Lambda=\left|\begin{array}{cccc}
a_{11} & a_{12} & \cdots & a_{1 m} \\
a_{21} & a_{22} & \cdots & a_{2 m} \\
\vdots & \vdots & & \vdots \\
a_{m 1} & a_{m 2} & \cdots & a_{m m}
\end{array}\right|
$$

invariant,${ }^{14}$ so

$$
\Lambda^{\prime}=\Lambda
$$

and thus

$$
\Omega=a_{1}^{\prime} a_{2}^{\prime} \cdots a_{m}^{\prime} c^{\prime} .
$$

It is seen in the present case, as with the fourdimensional case studied by Chisholm, ${ }^{14}$ that one does not need to know the individual $a_{j}^{\prime}$ of (32), only the product (38) that is completely determined by the known determinants, (35) and (36). The remaining quantity in (32) is given by these invariant determinants,

$$
c^{\prime}=\Omega / \Lambda \text {. }
$$

Thus the transformation that diagonalizes (28) never has to be explicitly calculated. One need only expand $\Omega$ by minors,

$$
\mathbf{\Omega}=C \Lambda+\sum_{i=1}^{m} \sum_{j=1}^{m} \mathbf{b}_{i} \cdot \mathbf{b}_{j}(-1)^{i+j+1} \Lambda_{i j},
$$

where $\Lambda_{i j}$ is $\Lambda$ with the $i$ th row and $j$ th column deleted. The final result (pending possible integrations of the $\rho$ 's) is

$$
\begin{aligned}
& S_{1 s_{1} \cdots i_{N}}^{\lambda_{1} \cdots \lambda_{N} \eta_{1} \cdots \eta_{M}}\left(\mathbf{p}_{1}, \mathbf{p}_{2}, \ldots, \mathbf{p}_{m} ; \mathbf{y}_{1}, \mathbf{y}_{2}, \ldots, \mathbf{y}_{N+M}\right) \\
& =\frac{\left(\lambda_{1} \cdots \lambda_{N}\right)^{5 / 2}}{2^{N} \pi^{N+M / 2-3 m / 2}} \\
& \quad \times \int_{0}^{\infty} d \rho_{1} \cdots d \rho_{N+M} \frac{\exp \left(-\lambda_{1}^{2} / 4 \rho_{1}-\cdots-\lambda_{N}^{2} / 4 \rho_{N}-\eta_{1}^{2} / 4 \rho_{N+1}-\cdots-\eta_{M}^{2} / 4 \rho_{N+M}\right)}{\left(\rho_{1} \cdots \rho_{N}\right)^{3 / 2}\left(\rho_{N+1} \cdots \rho_{N+M}\right)^{1 / 2}} \frac{e^{-\Omega / \Lambda}}{\Lambda^{3 / 2}}
\end{aligned}
$$

Note that numerical quadratures based on a Whittaker cardinal-sinc functions ${ }^{31}$ may be useful for these integrals.

\section{EXAMPLES}

In general, one will be left with the $N+M$ integrals of (41) to be evaluated numerically, although in specific cases one or more of these integrals may be done analytically. ${ }^{18,23}$ In the two integrals below, the known analytic result is found after integrating over the two-dimensional integral in (41).

Consider first the two-center integral

$$
S_{1 s}^{\lambda \lambda}\left(0 ; \mathbf{y}_{1}\right)=\int d^{3} x_{1} u_{1 s}^{\lambda}\left(\mathbf{x}_{1}-\mathbf{y}_{1}\right) V_{\lambda}\left(\mathbf{x}_{1}\right)
$$

Then

$$
\begin{aligned}
& \Lambda \equiv a_{11}=\rho_{1}+\rho_{2}, \\
& \mathbf{b}_{1}=\rho_{1} \mathbf{y}_{1},
\end{aligned}
$$

and

$$
C=\rho_{1} y_{1}^{2} .
$$

Defining $\Lambda_{11}=1$ for $m=1$ gives

$$
\Omega=\rho_{1} y_{1}^{2}\left(\rho_{1}+\rho_{2}\right)-\rho_{1}^{2} y_{1}^{2}=\rho_{1} \rho_{2} y_{1}^{2},
$$

so that

$$
S_{1 s}^{\lambda \lambda}\left(0 ; \mathbf{y}_{1}\right)=\frac{\lambda^{5 / 2}}{2} \int_{0}^{\infty} d \rho_{1} d \rho_{2} \frac{\exp \left[-\lambda^{2}\left(\rho_{1}+\rho_{2}\right) /\left(4 \rho_{1} \rho_{2}\right)-y_{1}^{2} \rho_{1} \rho_{2} /\left(\rho_{1}+\rho_{2}\right)\right]}{\rho_{1}^{3 / 2} \rho_{2}^{1 / 2}\left(\rho_{1}+\rho_{2}\right)^{3 / 2}} .
$$

Letting

$$
\tau=\rho_{2} /\left(\rho_{1}+\rho_{2}\right),
$$

the integral becomes ${ }^{32}$

$$
S_{1 s}^{\lambda \lambda}\left(0 ; \mathbf{y}_{1}\right)=\frac{\lambda^{5 / 2}}{2} \int_{0}^{1} d \tau \frac{1}{\tau^{1 / 2}} \int_{0}^{\infty} d \rho_{1} \frac{\exp \left(-\lambda^{2} / 4 \rho_{1} \tau-y_{1}^{2} \rho_{1} \tau\right)}{\rho_{1}^{5 / 2}}=\frac{\pi^{1 / 2}}{\lambda^{1 / 2}}\left(1+\lambda y_{1}\right) e^{-\lambda y_{1}}
$$


which matches the result of Shibuya and Wulfman. ${ }^{33}$

Next consider the Fourier transform of the product of one-center $1 s$ orbitals, with nonsymmetrical normalization,

$$
S_{1 s 1 s}^{\lambda \lambda}\left(\mathbf{p}_{1} ; 0\right)=\int d^{3} x_{1} e^{-i \mathbf{p}_{1} \cdot \mathbf{x}_{1}} u_{1 s}^{\lambda *}\left(\mathbf{x}_{1}\right) u_{1 s}^{\lambda}\left(\mathbf{x}_{1}\right) \text {. }
$$

In this case $C=0, \Lambda$ is given by (43), and

$$
\mathbf{b}_{1}=\frac{i \mathbf{p}_{1}}{2},
$$

so that

$$
\Omega=-b_{1}^{2}=\frac{p_{1}^{2}}{4} .
$$

Therefore

$$
S_{1 s 1 s}^{\lambda \lambda}\left(\mathbf{p}_{1} ; 0\right)=\frac{\lambda^{5}}{4 \sqrt{\pi}} \int_{0}^{\infty} d \rho_{1} d \rho_{2} \frac{\exp \left[-\lambda^{2}\left(\rho_{1}+\rho_{2}\right) /\left(4 \rho_{1} \rho_{2}\right)-p_{1}^{2} / 4\left(\rho_{1}+\rho_{2}\right)\right]}{\rho_{1}^{3 / 2} \rho_{2}^{3 / 2}\left(\rho_{1}+\rho_{2}\right)^{3 / 2}} .
$$

With $\tau$ as in (48),

$$
\rho_{1}=\rho_{2} \frac{(1-\tau)}{\tau} \text {, }
$$

so that the integral becomes ${ }^{32}$

$$
S_{1 s 1 s}^{\lambda \lambda}\left(\mathbf{p}_{1} ; 0\right)=\frac{\lambda^{5}}{4 \sqrt{\pi}} \int_{0}^{1} d \tau \frac{\tau}{(1-\tau)^{3 / 2}} \int_{0}^{\infty} d \rho_{2} \frac{\exp \left\{-\left[\lambda^{2} /(1-\tau)+\tau p_{1}^{2}\right] / 4 \rho_{2}\right\}}{\rho_{2}^{7 / 2}} .
$$

Changing variables to $\sigma=1 / \rho_{2}$ and using the relation

$$
\begin{aligned}
& \frac{\tau}{(1-\tau)^{3 / 2}} \frac{2^{5}}{\left[\lambda^{2} /(1-\tau)+\tau p_{1}^{2}\right]^{5 / 2}} \\
& \quad=-\left.\frac{2^{6}}{3} \frac{\partial}{\partial \alpha} \frac{1}{\left[\lambda^{2}+\alpha \tau(1-\tau)\right]^{3 / 2}}\right|_{\alpha=p_{1}^{2}}
\end{aligned}
$$

give the known result, ${ }^{6}$

$$
S_{1 s 1 s}^{\lambda \lambda}\left(\mathbf{p}_{1} ; 0\right)=\frac{(2 \lambda)^{4}}{\left(4 \lambda^{2}+p_{1}^{2}\right)^{2}}
$$

One may multiply this result by $(2 / \lambda)^{3 / 2} \sqrt{\pi}$ and then let $\lambda \rightarrow \lambda / 2$ to obtain the Fourier transform of a single $1 s$ orbital, ${ }^{4}$ with nonsymmetrical normalization,

$$
S_{1 s}^{\lambda}\left(\mathbf{p}_{1} ; 0\right)=(2 \pi)^{3 / 2} \frac{2 \sqrt{2} \lambda^{5 / 2}}{\pi\left(\lambda^{2}+p_{1}^{2}\right)^{2}}
$$

\section{EXCITED STATES}

Because the shape function $\left(22^{\prime}\right)$ for excited states is disconnected from the quadratic form $e^{-R^{2} \rho}$ in (21) that contains all spatial dependence, the orthogonal transformation of Sec. III may be utilized with no modification for excited $s$ states. Consider the general-state hydrogenic orbital,

$$
u_{n l m}^{\lambda}(\mathbf{R})=\lambda^{3 / 2} N_{n l} F_{n l}(2 \lambda R / n) Y_{l m}(\hat{R}),
$$

where $\lambda$ is given in (6),

$$
N_{n l}=\frac{2}{n^{2}} \sqrt{(n-l-1) !(n+l) !},
$$

and

$$
F_{n l}(2 \lambda R / n)=\sum_{s=0}^{n-l-1} \frac{(-1)^{s}(2 \lambda / n)^{s+l} R^{s+l} e^{-\lambda R / n}}{(n-l-1-s) !(2 l+1+s) ! s !} .
$$

Then the Gaussian shape function for the radial part of a general-state hydrogenic orbital is simply

$$
\begin{aligned}
f_{n l}^{\lambda}(\rho)=\sum_{s=0}^{n-l-1} & (-1)^{s} \frac{(\lambda / n)^{s+l}}{(n-l-1-s) !(2 l+1+s) ! s !} \\
& \times \frac{1}{2 \sqrt{\pi}} \frac{e^{-\lambda^{2} / 4 n^{2} \rho}}{\rho^{(s+l+2) / 2}} H_{s+l+1}\left(\frac{\lambda}{2 n \sqrt{\rho}}\right),
\end{aligned}
$$

where

$$
R \geq 0 \text { and } \lambda>0 .
$$

Then (62) with $l=0$, multiplied by $\lambda^{3 / 2} N_{n 0} Y_{00}$, may simply be substituted for the $1 s$ shape function

$$
f_{1 s}^{\lambda}(\rho)=\frac{\lambda^{5 / 2}}{2 \pi} \frac{e^{-\lambda^{2} / 4 \rho}}{\rho^{3 / 2}}
$$

in (41) for each such orbital.

Because the final number of integrals depends on the initial number of products, it is useful to transform together those products of orbitals and potentials that have the same coordinate dependence. In what follows, the 
compact notation

$$
u_{I}(\mathbf{R}) \equiv u_{n_{I} l_{I} m_{I}}^{\lambda_{I}}(\mathbf{R})
$$

will be used so that a product such as

$$
u_{I_{1}}\left(\mathbf{R}_{1}\right) \cdots u_{J_{1}}\left(\mathbf{R}_{1}\right)
$$

may be written without complicated indices. Similar simplifications in the indices of $\mathcal{P}, \phi, h, g$, and $S$ will be used. For the object function made up of the radial part of a product of orbitals and potentials

$$
\begin{aligned}
\varphi_{I J}^{K L j}(R)= & R^{j-1} e^{-\eta R} \\
& \times F_{n_{I} l_{I}}\left(2 \lambda_{I} R / n_{I}\right) \cdots F_{n_{J} l_{J}}\left(2 \lambda_{J} R / n_{J}\right),
\end{aligned}
$$

where $j$ may be 0 or 1 , the shape function is just

$$
\begin{aligned}
h_{I J}^{\eta j}(\rho)=\frac{\exp \left[-\left(\gamma_{I J}^{\eta}\right)^{2} / 4 \rho\right]}{2^{j} \sqrt{\pi}} \sum_{s_{I}=0}^{n_{I}-l_{I}-1}(-1)^{s_{I}} \frac{\left(\lambda_{I} / n_{I}\right)^{s_{I}+l_{I}}}{\left(n_{I}-l_{I}-1-s_{I}\right) !\left(2 l_{I}+1+s_{I}\right) ! s_{I} !} \\
\times \cdots \times \sum_{s_{J}=0}^{n_{J}-l_{J}-1}(-1)^{s_{J}} \frac{\left(\lambda_{J} / n_{J}\right)^{s_{J}+l_{J}}}{\left(n_{J}-l_{J}-1-s_{J}\right) !\left(2 l_{J}+1+s_{J}\right) ! s_{J} !} \\
\times \frac{H_{s_{I}+l_{I}+\cdots+s_{J}+l_{J}+j}\left(\gamma_{I J}^{\eta} / 2 \sqrt{\rho}\right)}{\rho^{\left(s_{I}+l_{I}+\cdots+s_{J}+l_{J}+1+j\right) / 2}},
\end{aligned}
$$

where

$$
\gamma_{I J}^{\eta}=\lambda_{I} / n_{I}+\cdots+\lambda_{J} / n_{J}+\eta,
$$

and

$$
\begin{aligned}
& R \geq 0 \text { and } \gamma_{I J}^{\eta}>0 \text { if } j=1 \\
& R>0 \text { and } \gamma_{I J}^{\eta} \geq 0 \text { if } j=0 .
\end{aligned}
$$

For products of states and potentials in $M$ groupings of a common coordinate, the analogue of (1) is

$$
\begin{aligned}
& S_{I_{1} J_{1} \cdots I_{M}^{J_{M}}}^{\eta_{1} j_{1} \cdots \eta_{M^{j}}}\left(\mathbf{p}_{1}, \mathbf{p}_{2}, \ldots, \mathbf{p}_{m} ; \mathbf{y}_{1}, \mathbf{y}_{2}, \ldots, \mathbf{y}_{M}\right) \\
& =\int d^{3} x_{1} \cdots d^{3} x_{m} e^{-i\left(\mathbf{p}_{1} \cdot \mathbf{x}_{1}+\cdots+\mathbf{p}_{m} \cdot \mathbf{x}_{m}\right)} \\
& \quad \times\left[u_{I_{1}}\left(\mathbf{R}_{1}\right) \cdots u_{J_{1}}\left(\mathbf{R}_{1}\right) V_{\eta_{1}}\left(\mathbf{R}_{1}\right)\right] \cdots\left[u_{I_{M}}\left(\mathbf{R}_{M}\right) \cdots u_{J_{M}}\left(\mathbf{R}_{M}\right) V_{\eta_{M}}\left(\mathbf{R}_{M}\right)\right] .
\end{aligned}
$$

Then if all $u$ 's are $s$ states,

$$
\begin{aligned}
& S_{I_{1}^{\prime} J_{1}^{\prime} \cdots I_{M}^{\prime} J_{M}^{\prime}}^{\eta_{1} j_{1} \cdots \eta_{M}^{j_{M}}}\left(\mathbf{p}_{1}, \mathbf{p}_{2}, \ldots, \mathbf{p}_{m} ; \mathbf{y}_{1}, \mathbf{y}_{2}, \ldots, \mathbf{y}_{M}\right)\left(\lambda_{I_{1}^{\prime}} \cdots \lambda_{J_{M}^{\prime}}\right)^{3 / 2}\left(N_{I_{1}^{\prime}} \cdots N_{J_{M}^{\prime}}\right) \\
&\left.=\frac{2^{j_{1}+\cdots+j_{M}} \pi^{(M-3 m) / 2}(4 \pi)^{\left(J_{1}^{\prime}+\cdots+J_{M}^{\prime}\right) / 2}}{\int_{0}^{\infty}} d \rho_{1} \cdots d \rho_{M} h_{I_{1}^{\prime} J_{1}^{\prime}}^{\eta_{1} j_{1}}\left(\rho_{1}\right) \cdots h_{I_{M}^{\prime} J_{M}^{\prime}}^{\eta_{M} \rho_{M}}\right) \frac{e^{-\Omega / \Lambda}}{\Lambda^{3 / 2}},
\end{aligned}
$$

where the primes indicate $s$ states, and $\Lambda$ and $\Omega$ are as before, given by (36) and (40).

Products of orbitals in which $l \neq 0$ are more difficult to treat. But if such orbitals come paired and summed over $m$, as is the case when matrix elements include projection operators, ${ }^{34}$ the extension is straightforward. Consider the combination

$$
\sum_{m=-l}^{l} u_{n l m}^{\lambda *}(\mathbf{R}) u_{n^{\prime} l m}^{\lambda^{\prime}}\left(\mathbf{R}^{\prime}\right)
$$

The sum over spherical harmonics in (68) may be written

$$
\sum_{m=-l}^{l} Y_{l m}^{*}(\hat{R}) Y_{l m}\left(\hat{R}^{\prime}\right)=\frac{2 l+1}{4 \pi} P_{l}\left(\frac{\mathbf{R} \cdot \mathbf{R}^{\prime}}{R R^{\prime}}\right),
$$

where the Legendre polynomial may be written as ${ }^{35}$

$$
P_{l}(z)=\frac{(2 l-1) ! !}{l !}\left(z^{l}-\frac{l(l-1)}{2(2 l-1)} z^{l-2}+\frac{l(l-1)(l-2)(l-3)}{2(4)(2 l-1)(2 l-3)} z^{l-4}-\cdots\right) .
$$


Note also that

$$
\left(\mathbf{R} \cdot \mathbf{R}^{\prime}\right)^{j}=\left.\frac{\partial^{j}}{\partial \tau^{j}} e^{-(1-\tau) \mathbf{R} \cdot \mathbf{R}^{\prime}}\right|_{\tau=1} .
$$

Then for any of the $M$ products that contain such a pair,

$$
\begin{aligned}
& \mathcal{P}_{I J, I^{\prime} J^{\prime}}^{\eta j, \eta^{\prime}}\left(\mathbf{R}, \mathbf{R}^{\prime}\right)=\sum_{m=-l}^{l}[\left.R^{j-1} e^{-\eta R} F_{n_{I} 0}\left(2 \lambda_{I} R / n_{I}\right) \cdots F_{n_{J-1} 0}\left(2 \lambda_{J-1} R / n_{J-1}\right) F_{n_{J} l}\left(2 \lambda_{J} R / n_{J}\right) Y_{l m}^{*}(\hat{R}) \sqrt{4 \pi}\right] \\
& \times\left[R^{\prime j^{\prime}-1} e^{-\eta^{\prime} R^{\prime} F_{n_{I}, 0}\left(2 \lambda_{I^{\prime}} R^{\prime} / n_{I^{\prime}}\right)}\right. \\
&\left.\times \cdots \times F_{n_{J^{\prime}-1} 0}\left(2 \lambda_{J^{\prime}-1} R^{\prime} / n_{J^{\prime}-1}\right) F_{n_{J^{\prime}} l}\left(2 \lambda_{J^{\prime}} R^{\prime} / n_{J^{\prime}}\right) Y_{l m}\left(\hat{R}^{\prime}\right) \sqrt{4 \pi}\right]
\end{aligned}
$$

where $j$ and $j^{\prime}$ may be 0 or 1 , one may define the shape functional, a differential operator that will ultimately act on $\Omega$ and $\Lambda$,

$$
\begin{aligned}
& g_{I J, I^{\prime} J^{\prime}}^{\eta j, j^{\prime}}\left[\rho, \rho^{\prime} ; \tau\right]=4 \pi \frac{(2 l-1) ! !}{l !} \frac{\exp \left[-\left(\gamma_{I J}^{\eta}\right)^{2} / 4 \rho\right]}{\sqrt{\pi}} \frac{\exp \left[-\left(\gamma_{I^{\prime} J^{\prime}}^{\eta^{\prime}}\right)^{2} / 4 \rho^{\prime}\right]}{\sqrt{\pi}} \sum_{s_{I}=0}^{n_{I}-1} \frac{(-1)^{s_{I}}\left(2 \lambda_{I} / n_{I}\right)^{s_{I}}}{\left(n_{I}-1-s_{I}\right) !\left(1+s_{I}\right) ! s_{I} !} \\
& \times \cdots \times \sum_{s_{J-1}=0}^{n_{J-1}-1} \frac{(-1)^{s_{J-1}}\left(2 \lambda_{J-1} / n_{J-1}\right)^{s_{J-1}}}{\left(n_{J-1}-1-s_{J-1}\right) !\left(1+s_{J-1}\right) ! s_{J-1} !} \\
& \times \sum_{s_{J}=0}^{n_{J}-l-1} \frac{(-1)^{s_{J}}\left(2 \lambda_{J} / n_{J}\right)^{s_{J}+l}}{\left(n_{J}-l-1-s_{J}\right) !\left(2 l+1+s_{J}\right) ! s_{J} !} \sum_{s_{I^{\prime}}=0}^{n_{I^{\prime}}-1} \frac{(-1)^{s_{I^{\prime}}}\left(2 \lambda_{I^{\prime}} / n_{I^{\prime}}\right)^{s_{I^{\prime}}}}{\left(n_{I^{\prime}}-1-s_{I^{\prime}}\right) !\left(1+s_{I^{\prime}}\right) ! s_{I^{\prime}} !} \\
& \times \cdots \times \sum_{s_{J^{\prime}}=0}^{n_{J^{\prime}-1}-1} \frac{(-1)^{s_{J^{\prime}-1}}\left(2 \lambda_{J^{\prime}-1} / n_{J^{\prime}-1}\right)^{s_{J^{\prime}-1}}}{\left(n_{J^{\prime}-1}-1-s_{J^{\prime}-1}\right) !\left(1+s_{J^{\prime}-1}\right) ! s_{J^{\prime}-1} !} \\
& \times \sum_{s_{J^{\prime}}=0}^{n_{J^{\prime}}-l-1} \frac{(-1)^{s_{J^{\prime}}}\left(2 \lambda_{J^{\prime}} / n_{J^{\prime}}\right)^{s_{J^{\prime}}+l}}{\left(n_{J^{\prime}}-l-1-s_{J^{\prime}}\right) !\left(2 l+1+s_{J^{\prime}}\right) ! s_{J^{\prime}} !} \\
& \times\left(\frac{H_{\sigma_{I J}+l+j}\left(\gamma_{I J}^{\eta} / 2 \sqrt{\rho}\right) H_{\sigma_{I^{\prime} J^{\prime}}+l+j^{\prime}}\left(\gamma_{I^{\prime} J^{\prime}}^{\eta^{\prime}} / 2 \sqrt{\rho^{\prime}}\right)}{(2 \sqrt{\rho})^{\sigma_{I J}+l+j+1}(2 \sqrt{\rho})^{\sigma_{I^{\prime} J^{\prime}}+l+j^{\prime}+1}} \frac{\partial^{l}}{\partial \tau^{l}}\right. \\
& -\frac{l(l-1)}{2(2 l-1)} \frac{H_{\sigma_{I J}+l+j+2}\left(\gamma_{I J}^{\eta} / 2 \sqrt{\rho}\right) H_{\sigma_{I^{\prime} J^{\prime}}+l+j^{\prime}+2}\left(\gamma_{I^{\prime} J^{\prime}}^{\eta^{\prime}} / 2 \sqrt{\rho^{\prime}}\right)}{(2 \sqrt{\rho})^{\sigma_{I J}+l+j+3}(2 \sqrt{\rho})^{\sigma_{I^{\prime} J^{\prime}}+l+j^{\prime}+3}} \frac{\partial^{l-2}}{\partial \tau^{l-2}} \\
& +\frac{l(l-1)(l-2)(l-3)}{2(4)(2 l-1)(2 l-3)} \\
& \left.\times \frac{H_{\sigma_{I J}+l+j+4}\left(\gamma_{I J}^{\eta} / 2 \sqrt{\rho}\right) H_{\sigma_{I^{\prime} J^{\prime}}+l+j^{\prime}+4}\left(\gamma_{I^{\prime} J^{\prime}}^{\eta^{\prime}} / 2 \sqrt{\rho^{\prime}}\right)}{(2 \sqrt{\rho})^{\sigma_{I J}+l+j+5}(2 \sqrt{\rho})^{\sigma_{I^{\prime} J^{\prime}}+l+j^{\prime}+5}} \frac{\partial^{l-4}}{\partial \tau^{l-4}}-\cdots\right),
\end{aligned}
$$

where

$$
\sigma_{I J}=s_{I}+\cdots+s_{J-1}+s_{J} .
$$

This shape functional will be substituted in (67) for any appropriate pair

$$
h_{I J}^{\eta j}(\rho) h_{I^{\prime} J^{\prime}}^{\eta^{\prime} j^{\prime}}\left(\rho^{\prime}\right)
$$

and

$$
(1-\tau) \mathbf{R} \cdot \mathbf{R}^{\prime}
$$

will be included in (25) so that the elements of (28), $a_{i j}, \mathbf{b}_{i}$, and $C$, have additional terms,

$$
a_{i j}=\sum_{k=1}^{N+M} \rho_{k} t_{k i} t_{k j}+\sum_{k=1}^{N+M} \sum_{k^{\prime}>k} \sigma_{k k^{\prime}} t_{k i} t_{k^{\prime} j},
$$




$$
\begin{aligned}
& \mathbf{b}_{i^{\prime}}=\frac{i \mathbf{p}_{i^{\prime}}}{2}+\sum_{k=1}^{N+M} \rho_{k} t_{k i^{\prime}} \sum_{j=1}^{N+M} u_{k j} \mathbf{y}_{j}+\sum_{k=1}^{N+M} \sum_{k^{\prime}>k} \sigma_{k k^{\prime}} t_{k i^{\prime}} \sum_{j=1}^{N+M} u_{k^{\prime} j} \mathbf{y}_{j}, \\
& C=\sum_{k=1}^{N+M} \sum_{j=1}^{N+M} \sum_{j^{\prime}=1}^{N+M} \rho_{k} u_{k j} u_{k j^{\prime}} \mathbf{y}_{j} \cdot \mathbf{y}_{j^{\prime}}+\sum_{k=1}^{N+M} \sum_{k^{\prime}>k} \sum_{j=1}^{N+M} \sum_{j^{\prime}=1}^{N+M} \sigma_{k k^{\prime}} u_{k j} u_{k^{\prime} j^{\prime}} \mathbf{y}_{j} \cdot \mathbf{y}_{j^{\prime}},
\end{aligned}
$$

where

$$
\sigma_{k k^{\prime}}=\left\{\begin{array}{l}
1-\tau_{k k^{\prime}} \text { if } k \text { and } k^{\prime} \text { are in the set of pairs in which } l \neq 0 \\
0 \text { otherwise }
\end{array}\right.
$$

\section{CONCLUSION}

The Gaussian transform has been used to analytically reduce the general set of multicenter integrals involving products of Coulomb or Yukawa potentials and plane waves with hydrogenic orbitals in $s$ states (and some states with $l \neq 0$ ). This one-dimensional integral transform thus accomplishes the same goal as previous methods using the four-dimensional Fourier-Feynman integral transformation, allowing all angular dependence to be written within a single quadratic form that may then be diagonalized. This alternative transformation has several advantages over the Fourier-Feynman transformation. The radial part of the Fourier transformed general-state hydrogenic wave function, ${ }^{4}$

$$
\widetilde{f}_{n l}(\mathbf{p})=\frac{(p / \lambda)^{l}}{\left[1+(p / \lambda)^{2}\right]^{l+2}} C_{n-l-1}^{l+1}\left(\frac{(p / \lambda)^{2}-1}{(p / \lambda)^{2}+1}\right) .
$$

includes $\lambda$ in the quadratic form containing $p^{2}$ so that derivatives with respect to $\lambda$, to remove the polynomial in $p^{2}$ in the numerator (after expansion of the Gegenbauer polynomial $C$ ), act on all expressions containing the quadratic form to be diagonalized. Thus all derivatives must be performed on the final reduced form for each specific application of the general result. In contrast, the Gaussian transform sequesters $\lambda$ from the quadratic form that contains the angular dependence to be diagonalized so that all such derivatives may be taken at the start [actually done more directly through generation of the shape function $\left(22^{\prime}\right)$ ] without acting on the quadratic form. Thus the final result is truly final for the general problem, apart from the algebra of computing the determinants, (36) and (40), in each individual application.

The Fourier-Feynman transform requires the introduction of a three-dimensional integral for each term in (1) that must be diagonalized and then integrated out (and the one-dimensional Feynman integral that allows the angular dependence to be summed) in addition to the spatial integrals that must be diagonalized and integrated out. In contrast, the one-dimensional Gaussian transform leads directly to functions of the angular dependence having arguments that are summable so that only the spatial integrals must be diagonalized and integrated out. Therefore the Fourier-Feynman transformation requires calculation of both $(N+M)$-dimensional determinants and $m$-dimensional determinants, whereas the Gaussian transformation requires only that the $m$-dimensional determinants be calculated. Both of these simplifications in the present technique lead to much simpler functions in the final integrand to be computed numerically and, hence, a savings of computer time. Finally, in cases where one is interested in only the analytic behavior of (1), such as in testing dispersion relations, ${ }^{36}$ the approximation methods of Polkinghorne and Screaton, ${ }^{37}$ to find the behavior of (1) near a surface of singularities, may be applied with more confidence. This is because the approximation, necessary because of their use of the Feynman transformation (9), of extending the upper bound of integration from 1 to infinity, is not needed in (41). The disadvantage of the present, analytically reduced, form (41) over that obtained through use of the FourierFeynman transformation ${ }^{12}$ is that the former integral is of one higher dimension than the latter if no further analytical reduction (by using a program such as MACSYMA, for instance) may be done.

\section{ACKNOWLEDGMENTS}

This work was done while the author was a guest scientist conducting research through the National Research Council-NASA Research Associateship Program. Computer costs were funded by NASA-RTOP No. 681-17010-01-08 (RTOP denotes "Research and Technology Operating Plan").
${ }^{*}$ Present address: Department of Physics, Cardwell Hall, Kansas State University, Manhattan, KS 66506.

${ }^{1}$ S. F. Boys, Proc. R. Soc. London, Ser. A 200, 542 (1950).

${ }^{2}$ The more recent articles and reviews on GTO's are K. Faegri and H. J. Speis, J. Chem. Phys. 86, 7035 (1987); N. Rosch, ibid. 86, 4038 (1987); E. R. Davidson and D. Feller, Chem. Rev. 86, 681 (1986); and R. Poirier, R. Kari, and I. G. Csizmadia, Handbook of Gaussian Basis Sets (Elsevier, New York, 1985).

${ }^{3}$ See, for instance, J. D. Jackson and H. Schiff, Phys. Rev. 89,
359 (1953); J. C. Straton and M. D. Girardeau, in Abstracts of the Fifteenth International Conference on the Physics of Electronic and Atomic Collisions, Brighton, 1987, edited by J. Geddes, H. B. Gilbody, A. E. Kingston, and C. J. Latimer (Queen's University, Belfast, 1987).

${ }^{4}$ B. Podolsky and L. Pauling, Phys. Rev. 34, 109 (1929).

${ }^{5}$ The number of references using this technique is too large to list here. Examples of papers in which Fourier (momentum) techniques are prominently mentioned are H. P. Trivedi and E. O. Steinborn, Phys. Rev. A 27, 670 (1983); B. R. Junker, J. 
Phys. B 13, 1049 (1980); C. Guidotti, G. P. Arrighini, and F. Marinelli, Theor. Chim. Acta 53, 165 (1979); J. Avery and P. Ormen, Int. J. Quantum Chem. 18, 953 (1980); J. Grotendorst and E. O. Steinborn, J. Comput. Phys. 61, 195 (1985); A. W. Niukkanen, J. Math. Phys. 25, 698 (1984). E. J. Weniger, ibid. 26, 276 (1985); A. K. Bhattacharya and S. C. Dhabal, J. Chem. Phys. 84, 1598 (1986); E. J. Weniger and E. O. Steinborn, ibid. 87, 3709 (1987); J. S. Alper, ibid. 55, 3780 (1971), Eq. (18) with $R_{2}=0$ as corrected by K. G. Kay and J. S. Alper, ibid. 56, 4243 (1972); E. J. Weniger and E. O. Steinborn, J. Chem. Phys. 78, 6121 (1983); E. Filter and E. O. Steinborn, Phys. Rev. A 18, 1 (1978), Eq. (2.14); A. W. Niukkanen, Int. J. Quantum Chem. 25, 941 (1984); 25, 957 (1984); Y. B. Band, Phys. Rev. A 8, 243 (1973); B. Talukdar, J. Dutta, and H. P. Chattopadhay, J. Phys. B 17, 3211 (1984).

${ }^{6}$ J. C. Straton, Phys. Rev. A 35, 2729 (1987).

${ }^{7}$ H. B. Dwight, Tables of Integrals and other Mathematical Data (Macmillan, New York, 1961), p. 225, No. 859.004.

${ }^{8}$ R. P. Feynman, Phys. Rev. 76, 769 (1949).

${ }^{9}$ S. S. Schweber, An Introduction to Quantum Field Theory (Harper and Row, New York, 1962), p. 913.

${ }^{10}$ Jack C. Straton, Phys. Rev. A (to be published).

${ }^{11}$ For $n=2$, a simpler form is given in C. J. Joachain, Quantum Collision Theory (North-Holland, Amsterdam, 1983), p. 678.

${ }^{12}$ Jack C. Straton, Phys. Rev. A (to be published).

${ }^{13}$ I. S. Gradshteyn and I. M. Ryzhik, Table of Integrals, Series, and Products (Academic, New York, 1980), p. 317, No. 3.381(4).

14J. S. R. Chisholm, Proc. Cambridge Philos. Soc. 48, 300 (1952).

${ }^{15} \mathrm{~W}$. Grobner and N. Hofreiter, Integraltafel Zweiter Teil Bestimmte Integrale (Springer-Verlag, Berlin, 1961), p. 66, Nos. 314.9a and 314.10a; I. S. Gradshteyn and I. M. Ryzhik, Ref. 13, p. 307, Nos. 3.325 and 3.321(3).

${ }^{16}$ R. Kikuchi, J. Chem. Phys. 22, 148 (1954), with $x=1$ and $\lambda \rightarrow x$.

${ }^{17}$ W. Grobner and N. Hofreiter, Ref. 15 , p. 66, No. 314.9c; I. S. Gradshteyn and I. M. Ryzhik, Ref. 13, p. 340, No. 3.471(9) and p. 317, No. 3.381(4) with $\rho \rightarrow 1 / \sigma$ for $x=0$.

${ }^{18}$ I. Shavitt and M. Karplus, J. Chem. Phys. 36, 550 (1962).
${ }^{19}$ W. Grobner and N. Hofreiter, Ref. 15 , p. 66 , No. 314.10 ; minus a derivative with respect to $\lambda$ of I. S. Gradshteyn and I. M. Ryzhik, Ref. 13, p. 307, Nos. 3.325 and 3.321(3).

${ }^{20}$ I. S. Gradshteyn and I. M. Ryzhik, Ref. 13, p. 1033, No. $8.950(1)$.

${ }^{21}$ I. S. Gradshteyn and I. M. Ryzhik, Ref. 13, p. 840, No. 7.386.

22J. P. Wright, Massachusetts Institute of Technology Solid State and Molecular Theory Group, Quarterly Progress Report No. 50, 1963 (unpublished), p. 35.

${ }^{23}$ I. Shavitt and M. Karplus, J. Chem. Phys. 43, 398 (1965), Eq. (66).

${ }^{24}$ F. R. Burden and R. M. Wilson, Adv. Phys. 21, 825 (1972), p. 841.

${ }^{25}$ W. J. Taylor, J. Math. Phys. 19, 52 (1978).

${ }^{26}$ D. M. Bishop and R. L. Somorjai, J. Math. Phys. 11, 1150 (1970).

${ }^{27}$ A. Erdelyi, W. Magnus, F. Oberhettinger, and F. G. Tricomi, Tables of Integral Transforms (McGraw-Hill, New York, 1954), Vol. 1.

${ }^{28} \mathrm{~F}$. Oberhettinger and L. Badii, Tables of Laplace Transforms (Springer-Verlag, New York, 1973).

${ }^{29} \mathrm{G}$. E. Roberts and H. Kaufman, Table of Laplace Transforms (Saunders, Philadelphia, 1966).

${ }^{30}$ A. Erdelyi, W. Magnus, F. Oberhettinger, and F. G. Tricomi, Ref. 27, p. 246, No. 8, where $H_{n}(x) \equiv 2^{n / 2} H e_{n}(\sqrt{2} x)$.

${ }^{31}$ R. N. Hill (private communication); F. Stenger, SIAM (Soc. Ind. Appl. Math.) Rev. 23, 165 (1981).

${ }^{32}$ I. S. Gradshteyn and I. M. Ryzhik, Ref. 13, p. 967, No. 8.468 and p. 340, No. 3.471(9).

${ }^{33}$ T. Shibuya and C. C. Wulfman, Proc. R. Soc. London 286, 376 (1965).

34 J. C. Straton, Phys. Rev. A 35, 3725 (1987); M. D. Girardeau, ibid. 26, 217 (1982), and references therein.

${ }^{35}$ I. S. Gradshteyn and I. M. Ryzhik, Ref. 13, p. 1025, No. 8.911(1).

${ }^{36}$ A. K. Bhatia and A. Temkin, Phys. Rev. A 37, 1415 (1988).

${ }^{37}$ J. C. Polkinghorne and G. R. Screaton, Nuovo Cimento 15, 925 (1960). 